\title{
Numerical Investigation of the Fluids Mixing Efficiency in the Micromixer with Cylindrical Section of Swirl Flow
}

\author{
Lobasov A.S. ${ }^{1,2}$, Shebeleva A.A. ${ }^{1,2, a)}$, Shebelev A.V. ${ }^{1,2}$, Minakov A.V. ${ }^{1,2}$ \\ ${ }^{I}$ Siberian Federal University, \\ Svobodny av., 79, Krasnoyarsk, 660041, Russia \\ ${ }^{2}$ Kutateladze Institutes of Thermophysics, \\ Lavrentiev av., 1, Novosibirsk, 630090, Russia \\ ${ }^{a)}$ Corresponding author: an_riv@mail.ru
}

\begin{abstract}
A computational study of the micromixer form influence on the mixing efficiency and pressure drop in a wide range of Reynolds numbers $1 \leq \mathrm{Re} \leq 300$ was carried out. It was found that as the number of segments increases the pressure drop and mixing efficiency also increase in a whole range of Reynolds numbers. The obtained results were compared with a one for the T-type micromixer with direct mixing channel of the corresponding length. The dependence of the relative reduced mixing efficiency on the Reynolds number was obtained. It is revealed that the use of micromixers with a complex geometric shape allows to intensify the mixing processes, especially at low Reynolds numbers.
\end{abstract}

\section{INTRODUCTION}

The micromixer is one of the main components in microfluidic systems, which are widely used in biomedical analysis and chemical synthesis. The flow in the microchannels is usually laminar and mixing between different phases is largely depended on mass diffusion. Because of this, over the past two decades, various ways to increase the mixing productivity have been considered [1,2]. Active micromixers usually produce good mixing, but their manufacturing cost is high, and integration with other devices is relatively more complex. Accordingly, passive micromixers are preferred in many situations, especially for single use. While active micromixers usually require external power sources to improve mixing, the microchannels with a specific geometry must be used to increase mixing performance in passive micromixers. Most of the proposed passive micromixers have shown good efficiency, especially in applications with fairly high Reynolds numbers.

In applications related to chemical or biochemical reactions, for example, micro-scale total analysis systems (mTAS), rapid mixing at a low flow rate $(\operatorname{Re}<10)$ is desirable. The designs that using patterned grooves at the bottom of the channel [3], the split-and-recombine (SAR) systems [4, 5], three-dimensional (3D) topological structures [6], chains of repeating segments with the profiles of arbitrary design [7] or channels with a two-layer intersection [8] work well at low Reynolds numbers. Spiral flows formed by patterned grooves [3] or repeating segments with the profiles of arbitrary design [7] can increase the area of the phase boundary and, thus, facilitate mixing. The advection caused by a combination of SAR and 3D serpentine channels contributes to a significant improvement in fluid mixing [4-10]. Micromixers with crossed obstacles inside the microchannel and chain mixers with microstructures located on the upper and lower walls of microchannels allow achieving high mixing efficiency at low Reynolds numbers [11, 12]. In addition, a micromixer based on crosslinked double spiral tubes was proposed for quick mixing at low Reynolds numbers [13]. But most of the proposed designs complicate the mixing channel and increase the complexity of its manufacture. So, finding the ways to increase the mixing performance is necessary for many areas of science and technology.

To reach this goal, a channel with such shape that allows reducing the fluids mixing path and increasing the contact area has been reviewed it this paper. A computational study of fluids mixing efficiency in micromixers with complex geometric shapes with different number of segments (two, four or six) has been carried out. The micromixer consists of inlet channels of 200x200x3400 $\mu \mathrm{m}$ in size and a mixing channel with different number of 
segments. A straight section of 200x400x700 $\mu \mathrm{m}$ and a cylindrical section with a diameter of $400 \mu \mathrm{m}$ and a height of $800 \mu \mathrm{m}$ were considered as one segment. The obtained results were compared with direct T-type channels of corresponding length: $5891.5 \mu \mathrm{m}$ for two segments; $11083 \mu \mathrm{m}$ for four segments; $16274.5 \mu \mathrm{m}$ for six segments.

Pure water with a density of $1000 \mathrm{~kg} / \mathrm{m}^{3}$ and a viscosity of $0.001 \mathrm{~Pa} \cdot \mathrm{s}$ was supplied to one of the inlets. Water with same properties, but tinted with paint to indicate the mixing process, was supplied to the other inlet of micromixer. The paint was considered as a passive scalar, its diffusion coefficient in water was $2.63 \cdot 10^{-10} \mathrm{~m}^{2} / \mathrm{s}$. Reynolds numbers ranged from $1 \leq \operatorname{Re} \leq 300$.

\section{MATHEMATICAL METHOD}

In this work were considered incompressible flows of multi-component fluids, which are described using a hydrodynamic approach based on the solution of the Navier-Stokes equations:

$$
\frac{\partial \rho}{\partial t}+\nabla(\rho \mathbf{v})=0, \quad \frac{\partial \rho \mathbf{v}}{\partial t}+\nabla(\rho \mathbf{v v})=-\nabla p+\nabla \mathbf{T},
$$

where $\rho$ is the fluid density, $p$ is the pressure, $\mathbf{v}$ is the velocity, and $\mathbf{T}$ is the tensor of viscous stresses, which components are determined as:

$$
\mathbf{T}_{i j}=\mu \cdot\left(\frac{\partial u_{j}}{\partial x_{i}}+\frac{\partial u_{i}}{\partial x_{j}}\right) .
$$

Here $\mu$ is the mixture viscosity and $u_{i, j}$ are the velocity vector components.

The effective viscosity and the density of the mixture are determined through the components mass fraction $f$ and their effective viscosities $\mu_{1}, \mu_{2}$ and partial densities $\rho_{1}, \rho_{2}$ respectively:

$$
\mu=f \mu_{1}+(1-f) \mu_{2}, \quad \frac{1}{\rho}=\frac{f}{\rho_{1}}+\frac{(1-f)}{\rho_{2}} .
$$

The evolution of mass concentrations is determined by the next equation:

$$
\frac{\partial \rho f}{\partial t}+\nabla(\rho f \mathbf{v})=\nabla(\rho D \nabla f)
$$

where $D$ is the diffusion coefficient.

The software package for computational fluid dynamics "Ansys Fluent" was used to solve the described above equations system.

The quantitative characteristic of mixing efficiency is the parameter $M=1-\sqrt{\sigma / \sigma_{0}}$, where $\sigma=V^{-1} \int_{V}(f-\langle f\rangle)^{2} d V$ is the root-mean-square deviation of the mass fraction of mixture component $f$ from its average value $\langle f\rangle, \sigma_{0}=\langle f\rangle \cdot(1-\langle f\rangle)$ is the maximum root-mean-square deviation, and $V$ is the volume of the computational domain. Calculation was carried out on a polyhedral mesh with a mesh size of $10^{-5} \mathrm{~m}$. Methodological calculations showed that such detailing of mesh is sufficient to resolve interface between two miscible media. Convective terms of transport equations are approximated using second-order upwind schemes QUICK and TVD. The Neumann conditions were specified at outlet of mixing channel. No-slip conditions were set on channel walls.

\section{RESULTS AND DISCUSSION}

A computational study of the segments number effect on mixing regimes, as well as on the mixing efficiency and pressure drop was carried out. The results of numerical simulations of two fluids mixing are presented as velocity streamlines (figs. 1, 3, 5 and 6) and as distribution of concentration field over cross-sections of micromixer (figs. 2 and 4) for Reynolds numbers equal to 120 and 180. In the last figures, the red colour corresponds to the maximum concentration of the first fluid, the blue colour corresponds to the maximum concentration of the second fluid, the green colour corresponds to $50 \%$ concentration of both fluids, i.e. their complete mixing.

As one can see in Figure 1, an unmixed stream enters to the first cylinder, where it swirls in a spiral, and then it enters to the second cylinder, where it is again swirls and mixes. It can be seen, that two main vortex structures are formed. The first vortex propagates along the axis of cylinders (fig. 1b) and the second one can be found in the 
upper right corner of the first cylinder and lower right corner of the second cylinder (fig. 1a). Figure 2 represents the mixing process of fluids in four vertical cross-sections. Vertical coordinate ranges from $0 \mu \mathrm{m}$ to $800 \mu \mathrm{m}$. Such process also occurs in other cases, however, for $\mathrm{Re}=180$ a well-mixed flow enters to the first cylinder, because of an engulfment flow regime begins in $\mathrm{T}$-shaped micromixers of such sizes already at $\mathrm{Re}=145$. Comprehensive investigations of mixing regimes and efficiency of T-shaped mixers, as well as the effect of the engulfment regime on these parameters, were carried out in $[14,15]$. It was found, that mixing efficiency and pressure drop increase in the whole investigated range of Reynolds numbers. It can be seen in Figure 7a. It also clearly seen in this figure that the dependence of mixing efficiency from Reynolds number is non-linear. Moreover, several different regimes can be distinguished, depending on the angle of the curve in this graph. In $[14,15]$ were determined six different mixing regimes that occur in T-shaped micromixer depending on the Reynolds number. Analyzing these papers, graph in Figure 7a and Figures 1-4 it can be concluded, that the same regimes occur in the straight section of the first segment of micromixer and influence on the mixing efficiency of such complex micromixer.
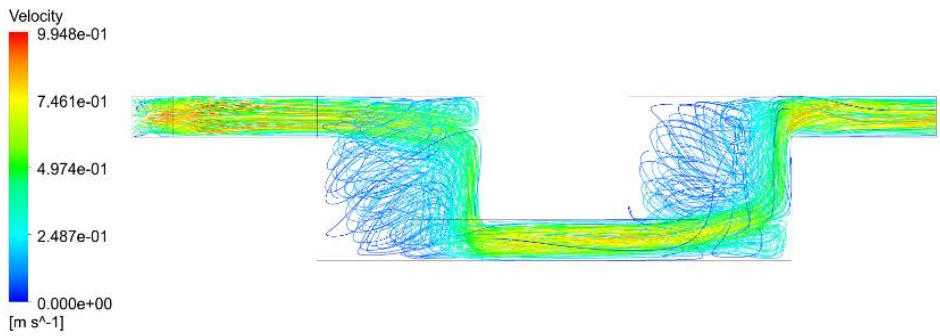

(a)

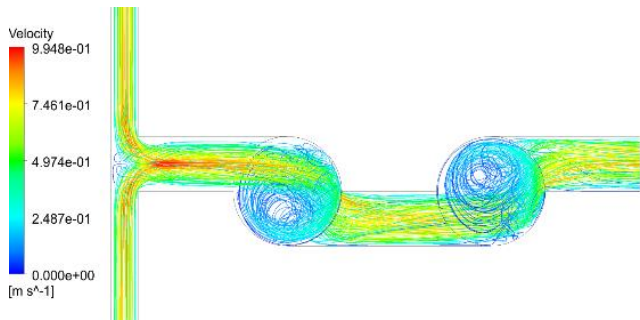

(b)

FIGURE 1. Velocity streamlines: a) side view b) top view. Two segments, $R e=120$.

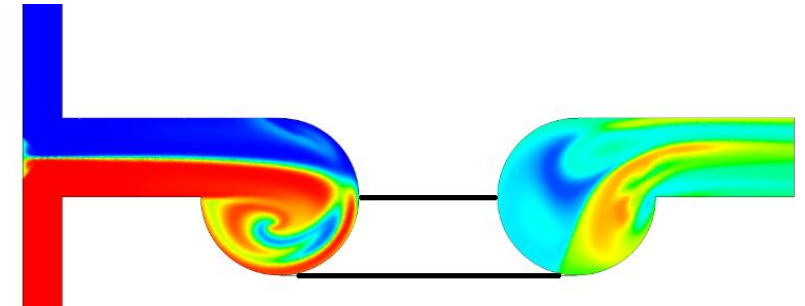

a)

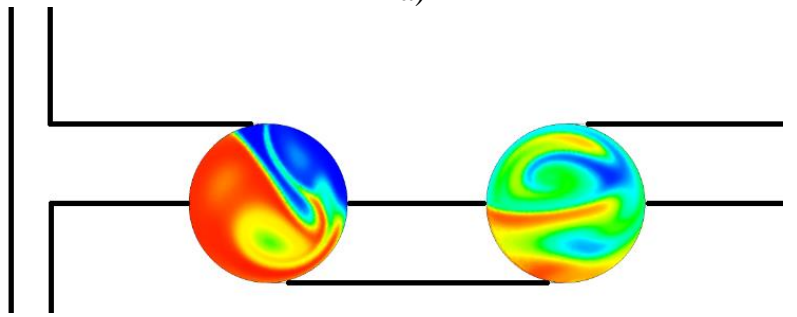

c)

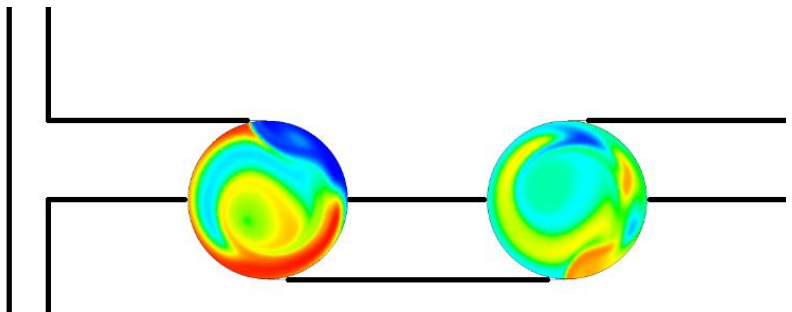

b)

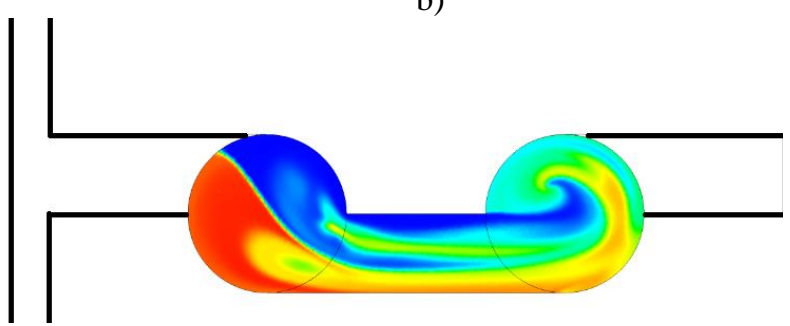

d)

FIGURE 2. Concentrations distribution in the cross-sections at different distances from bottom wall of the mixer: a) $700 \mu \mathrm{m}$; b) $400 \mu \mathrm{m}$; c) $200 \mu \mathrm{m}$ and d) $100 \mu \mathrm{m}$. Two segments, $R e=120$.

Next, the effect of segments number on the mixing efficiency and pressure drop of micromixer was investigated. It was found that that two main vortex structures, described above, are formed in every cylindrical section regardless of the number of the segments or Reynolds number. It can be seen in Figures 5, 6, where the velocity streamlines represented in the micromixers with six segments at Reynolds numbers equal to 120 and 180. It was established that in the entire range of Reynolds numbers mixing efficiency of a micromixer with six segments was higher, than the same value for the mixer with two and four segments. So, at $\mathrm{Re}=50$ the mixing efficiency for six segments was 1.25 times higher, than for four segments and 2.02 times higher, than for two segments. At $\operatorname{Re}=160$ the mixing efficiency for six segments was 1.11 times higher, than for four segments and 1.42 times higher, than for two segments. This can be seen from fig. 7a. Besides, it was found that pressure drop in the micromixer with six segments was higher than the same value for the mixer with two and four segments over the entire range of Reynolds numbers (fig. 7b). 


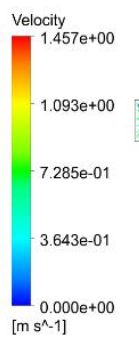

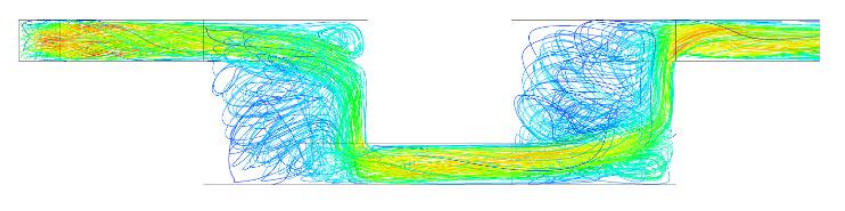

(a)

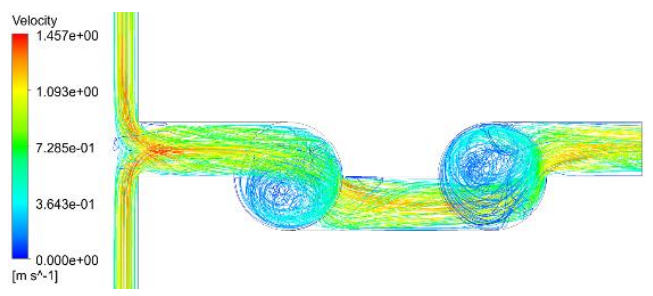

(b)

FIGURE 3. Velocity streamlines: a) side view b) top view. Two segments, $R e=180$.

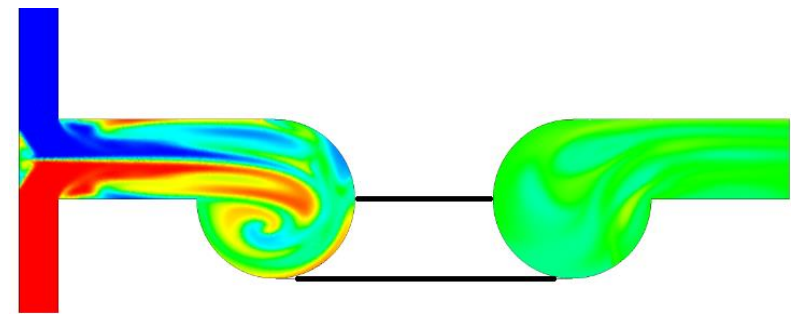

a)

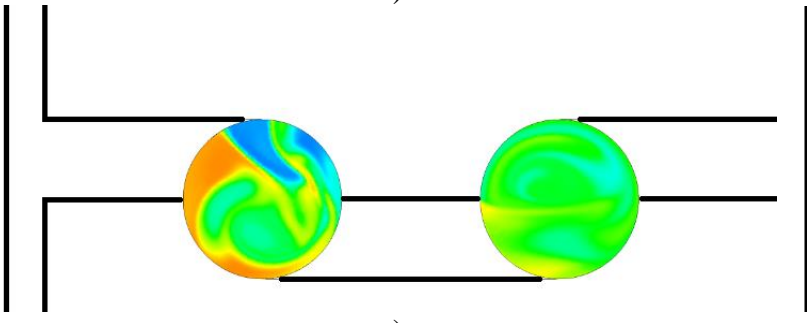

c)

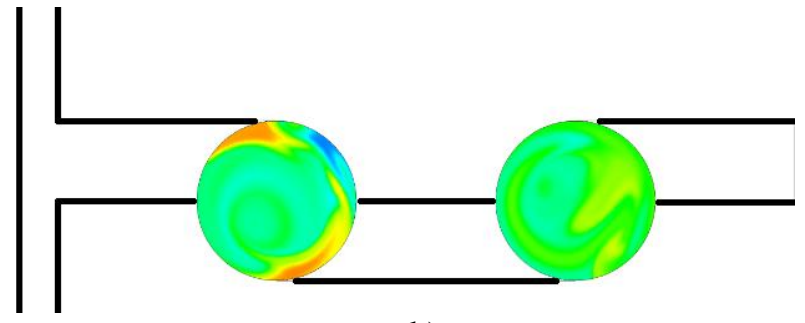

b)

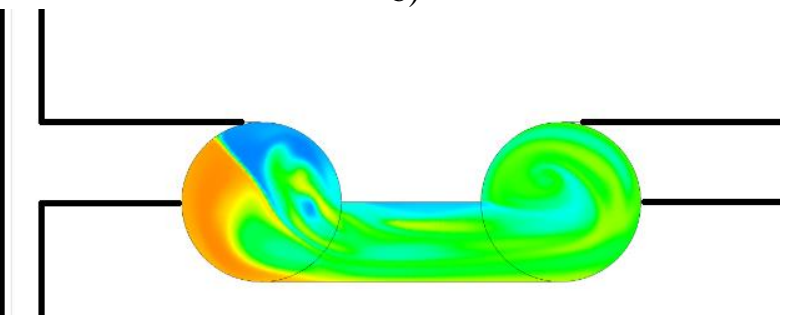

d)

FIGURE 4. Concentrations distribution in the cross-sections at different distances from bottom wall of the mixer: a) $700 \mu \mathrm{m}$; b) $400 \mu \mathrm{m}$; ) $200 \mu \mathrm{m}$ and d) $100 \mu \mathrm{m}$. Two segments, $\operatorname{Re}=180$.

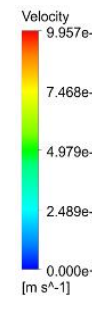

(a)

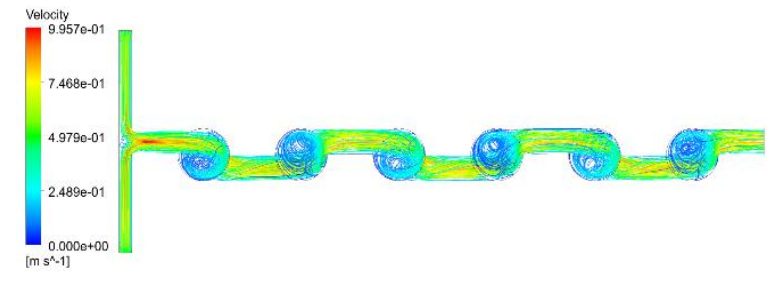

(b)

FIGURE 5. Streamline of velocity: a) side view b) top view for $6 \mathrm{BE}$ and $\mathrm{Re}=120$.

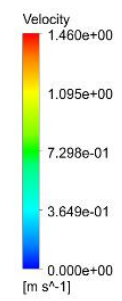

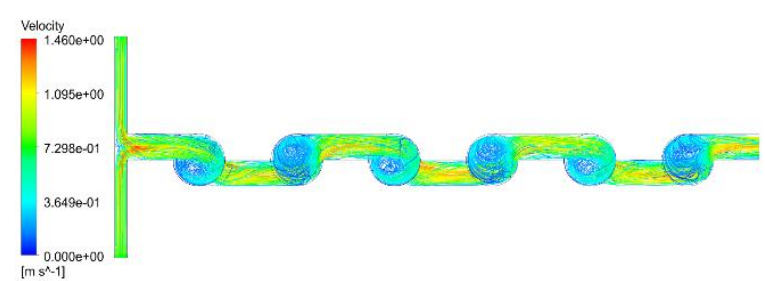

(b)

FIGURE 6. Streamline of velocity: a) side view b) top view for $6 \mathrm{BE}$ and $\mathrm{Re}=180$. 
At $\mathrm{Re}=100$ the pressure drop for the mixer with six segments was 1.44 times higher, than with four segments and 2.57 times higher, than with two segments. At $\mathrm{Re}=250$ the pressure drop mixer with six segments was 1.45 times higher, than with four segments and 2.66 times higher, than with two segments. It is shown that an increase in the number of the segments increases both pressure drop and mixing efficiency of the micromixer over the entire range of Reynolds numbers $1 \leq \operatorname{Re} \leq 300$. It follows that it is necessary to use such a value as reduced mixing efficiency, which determined as the ratio of mixing efficiency to pressure drop. But, often, using even such a value is not enough to show an increase in the efficiency of the micromixer compared to a straight $\mathrm{T}$-shaped mixer. Therefore, in this paper, it is proposed to use relative reduced mixing efficiency, which determined as the ratio of reduced mixing efficiency of considered micromixer to reduced mixing efficiency of straight T-shaped mixer.

The dependences of relative reduced mixing efficiency on Reynolds number for micromixers with different number of segments were obtained (see fig. 8). As can be seen in that picture, the reduced mixing efficiency of complex geometry mixer was higher, than the same value for straight $\mathrm{T}$-channels of the corresponding length in considered range of Reynolds numbers $1 \leq \operatorname{Re} \leq 300$. In particular, in the region of Reynolds numbers $1 \leq \operatorname{Re} \leq 140$, that value was the highest for the geometry with two segments and the lowest for the geometry with six segments. In the range of Reynolds numbers $145 \leq \operatorname{Re} \leq 200$, in contrast, it was observed the opposite situation, when relative reduced mixing efficiency shows the highest values for the geometry with six segments and the lowest for the geometry with two segments. In the range of Reynolds numbers $200 \leq \mathrm{Re} \leq 300$, it was found, that again relative reduced mixing efficiency was the highest for the geometry with two segments and the lowest for the geometry with six segments. It can be explained by the simultaneous influence of changes in flow regimes with an increase in the Reynolds number and the number of segments. As one can see, in some cases, the restructuring of the flow regime is more strongly affected in the relative reduced mixing efficiency, and in others cases, an increase in the number of segments has a stronger effect.

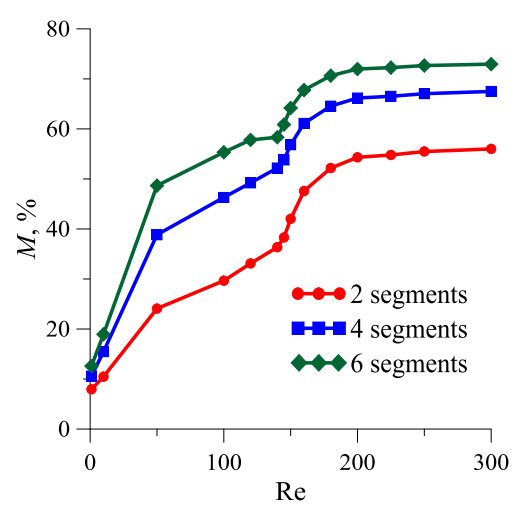

(a)

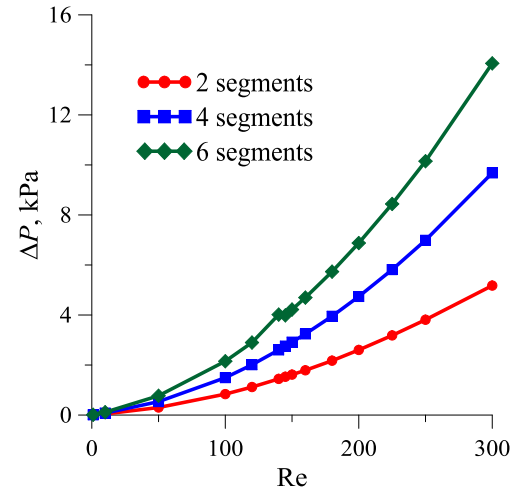

(b)

FIGURE 7. The dependences of mixing efficiency (a) and pressure drop (b) on the Reynolds number for the micromixers with different number of segments
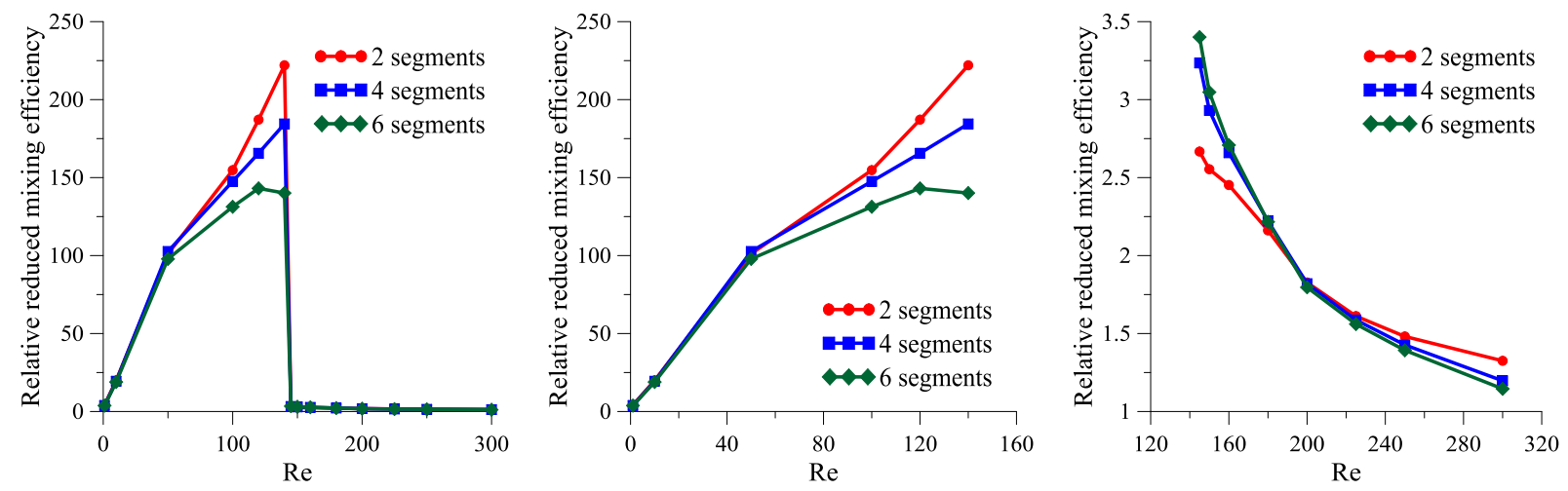

FIGURE 8. The dependence of the relative reduced mixing efficiency on the Reynolds number for micromixers with different number of segments 


\section{SUMMARY}

A computational study of the micromixer geometry effect on the mixing efficiency and pressure drop of such mixer in a wide range of Reynolds numbers $1 \leq R e \leq 300$ was carried out. It was found that as the number of segments increases the pressure drop and mixing efficiency increase too. The obtained results were compared with a one for the T-type micromixer of the corresponding length. It was established that it is necessary to use reduced mixing efficiency, which determined as the ratio of mixing efficiency to pressure drop. But, often, using such a value is not enough to show an increase in the efficiency of the micromixer. Therefore, in this paper, it was proposed to use relative reduced mixing efficiency, which determined as the ratio of reduced mixing efficiency of considered micromixer to reduced mixing efficiency of straight $\mathrm{T}$-shaped mixer. The dependence of the relative reduced mixing efficiency on the Reynolds number was obtained. It was shown that the relative reduced mixing efficiency of complex geometry mixer was higher than one in considered range of Reynolds numbers $1 \leq \operatorname{Re} \leq 300$. It is revealed that the use of micromixers with a complex geometric shape allows to intensify the mixing processes, especially at low Reynolds numbers, for example at $\mathrm{Re}=140$ this value about 230 .

\section{ACKNOWLEDGMENTS}

The reported study was funded by Russian Foundation for Basic Research, Government of Krasnoyarsk Territory, Krasnoyarsk Regional Fund of Science to the research project No 18-48-243011: "The calculation study of ways to enhance the efficiency of microfluidic reactors".

\section{REFERENCES}

1. N.-T. Nguyen, Z. Wu. J. Micromech. Microeng. 15, R1-R16 (2005).

2. C.-Y. Lee, W.-T. Wang, C.-C. Liu, L.-M. Fu. Chem. Eng. J. 288, 146-160 (2016).

3. A.D. Stroock, S.K.W. Dertinger, A. Ajdari, I. Mezic, H.A. Stone, G.M. Whitesides. Science 295, 647-651 (2002).

4. F. Schonfeld, V. Hessel, C. Hofmann. Lab. Chip 4, 65-69 (2004).

5. D.S. Kim, S.H. Lee, T.H. Kwon, C.H. Ahn. Lab. Chip 5, 739-747 (2005).

6. H. Chen, J.-C. Meiners. Appl. Phys. Lett. 84, 2193-2195 (2004).

7. C. Simonnet, A. Groisman. Phys. Rev. Lett. 94, 134501 (2005).

8. H.M. Xia, S.Y.M. Wan, C. Shu, Y.T. Chew. Lab. Chip. 5, 748-755 (2005).

9. $\quad$ S.W. Lee, S.S. Lee. Sens. Actuator. B. Chem. 129, 364-371 (2008).

10. J.J. Chen, Y.R. Lai, R.T. Tsai, J.D. Lin, C.-Y. Wu. Chem. Eng. Sci. 66, 2164-2176 (2011).

11. W.-S. Yoo, J.S. Go, S. Park, S.-H. Park. J. Micromech. Microeng. 22, 1-9 (2012).

12. V. Viktorov, M. Nimafar. J. Micromech. Microeng. 23, 1-13 (2013).

13. K. Liu, Q. Yang, F. Chen, Y. Zhao, X. Meng, C. Shan, Y. Li. Microfluid. Nanofluid. 19, 169-180 (2015).

14. A.S. Lobasov, A.V. Minakov, Chem. Eng. \& Proces.: Process Intensification 124, 11-23 (2018).

15. A.S. Lobasov, A.V. Minakov, V.V. Kuznetsov, V.Y. Rudyak, A.A. Shebeleva, Chem. Eng. \& Proces.: Process Intensification 134, 105-114 (2018). 